\title{
Wie Kooperation gelingen kann
}

\section{Der "Herforder Weg" bei der Zusammenarbeit von Jugendamt und Schule}

ANDREAS SPILKER

Andreas Spilker leitet das Jugend-

amt der Stadt Herford.

andreas.spilker@herford.de

\author{
Kinder, Jugendliche und ihre Familien benötigen \\ vielfach Hilfe und Unterstützung bei Problemen, die \\ sowohl die Schule als auch die Jugendhilfe betreffen. \\ In Herford beschreiten beide Institutionen deshalb \\ einen gemeinsamen Weg, um angemessene Hilfen \\ zu finden - ohne dass die Letztverantwortung \\ des jeweiligen Bereichs in Frage gestellt wird.
}

Die Zusammenarbeit zwischen Jugendhilfe und Schule ist für jede Kommune eine zukunftsweisende Aufgabe. Die grundsätzliche gesetzliche Verankerung dieser Zusammenarbeit ist für die Jugendhilfe in $\mathbb{S} 81$ SGB VIII sowie für das Bundesland Nordrhein-Westfalen in $\mathbb{} 7$ Kinder- und Jugendförderungsgesetz NRW geregelt. Für die Schulseite findet sich eine Verpflichtung in \5 Schulgesetz NRW. In dieser Zusammenarbeit geht es um die Verknüpfung von zwei sehr verschiedenen Systemen und besonders um die Schaffung tragfähiger Strukturen.

Vor diesem Hintergrund der gesetzlichen Vorgaben sowie eines zunehmenden ganzheitlichen Bildungsverständnisses, einer deutlich höheren Bedeutung der vorschulischen Bildungsförderung und des damit verbundenen gesetzlich geregelten Rechtsanspruches auf einen Betreuungsplatz ab August 2013, der Ganztagsschulentwicklung und dem Schutzauftrag von Jugendhilfe und Schule über den $\mathbb{\int} 8$ a SGB VIII und \$ 42 Abs. 6 Schulgesetz NRW haben sich in den letzten Jahren auch in Herford die Strukturen für die Kooperation von Jugendhilfe und Schule (weiter)entwickelt und stabilisiert.

Die Jugendhilfe muss ein besonderes Interesse daran haben, gerade die sensiblen Bereiche der Übergänge im Bereich der Kita zur Grundschule, von der Grundschule in die weiterführenden Schulen und von der weiterführende Schule in die Berufswelt zu begleiten.
Gerade bei Kindern und Jugendlichen mit besonderen Unterstützungsbedarf kann es in den Übergangssituationen zu Krisen kommen, die eine erfolgreiche Stabilisierung wieder gefährden und oft zu ergänzenden Hilfen zur Erziehung führen. Die Übergänge sind im Rahmen der Jugendhilfe auch deshalb so wichtig, da Kontinuitäten bei der Entwicklung von Kindern und Jugendlichen sehr stark wirken, durch die Übergänge von unterschiedlichen Regeleinrichtungen kommt es im Bildungssystem jedoch zu Brüchen.

\section{Förderangebote bis zum 27. Lebensjahr}

Die Stadt Herford ist in den letzten Jahren mit ihrem »Herforder Weg « - einem Weg von Förder- und Unterstützungsmöglichkeiten beginnend vor der Geburt eines Kindes bis zum 27. Lebensjahr - auch wichtige Schritte in Richtung Zusammenarbeit bei den Übergängen auch im Bereich Jugendhilfe und Schule gegangen und wir wollen den eingeschlagen Weg weiter verfolgen.

Der Allgemeine Soziale Dienst wurde schon in den neunziger Jahren organisatorisch an den Grundschulbezirken ausgerichtet, so dass jede Schule einen festen Ansprechpartner im Jugendamt hatte. Auch richtete die Stadt Herford drei Teilzeitstellen für Schulsozialarbeiterinnen an einer Haupt- und Förderschule ein und unterstützte schulbe- 
zogene Projekte mit Mitteln der Erziehungshilfe und der Jugendförderung.

Zunächst waren die Schulsozialarbeiterinnenstellen an das Jugendamt organisatorisch angegliedert. Es wurde jedoch deutlich, dass das Schulsystem tragend in der Umsetzung der Aufgabenwahrnehmung war, sodass nun die Teamanbindung an die Abteilung Bildung erfolgte.

Die seit Jahren bestehende gute, intensive Zusammenarbeit zwischen den elf Herforder Grundschulen und dem Allgemeinen Sozialen Dienst führten zu gemeinsamen Projekten, insbesondere bei der Vernetzung von pädagogischen Angeboten der Hilfen zur Erziehung, den Angeboten des offenen Ganztags und der schulischen Angebote. Im Jahr 2006 nahmen die beteiligten Kooperationspartner an dem Projekt des Landesjugendamtes »Integration flexibler Erziehungshilfen in die offene Ganztagsschule « teil. Die Ergebnisse dieser Projektteilnahme führten zu ersten Festlegungen von verbindlichem gemeinsamem Vorgehen, um den Unterstützungsbedarf eines Kindes oder einer Familie zu erkennen sowie Unterstützung zu planen und zu realisieren.

\section{Ein runder Tisch von Jugendamt und Schulsozialarbeit}

Durch die aktive Mitarbeit des Jugendamtes an den Planungen und bei der Umsetzung eines neu einzurichtenden »Kompetenzzentrum für sonderpädagogischer Förderung« im Zusammenhang der Einschätzung nach Bedarfen der Eingliederungshilfe für seelisch behinderte Kinder und Jugendliche $(\mathbb{S} 35 \mathrm{a}$ SGB VIII).

Auf der Basis dieser guten Zusammenarbeit befinden wir uns zur Zeit unter dem Motto »Ungleiches ungleich behandeln« konkret mit vier Herforder Grundschulen aus besonders belasteten Sozialräumen in Planungsgesprächen, um vier Projektansätze der weiteren Zusammenarbeit zu entwickeln - auch um zu weiteren wirksamen Steuerungsansätzen für die Ausgestaltung der Hilfen zur Erziehung zu gelangen. Die vier Projektansätze im Einzelnen sind:

- Entwicklung einer Generalvereinbarung als Muster der Zusammenarbeit zwischen Jugendhilfe und Schule für die Umsetzung des gesetzlichen Auftrages nach dem Bundeskinderschutzgesetz

- Entwicklung einer sozialräumlichen verbindlichen Zusammenarbeit zwischen den Angeboten der Grundschule, der Offenen Ganztagsschule, des Jugendamtes mit den Schwerpunkten der Jugendsozialarbeit und der Hilfen zur Erziehung

- Weiterentwicklung von »Lebenswelten im Sozialraum " - der Entwicklung eines vernetzten Angebotes von Grundschule, Jugendsozialarbeit und Bildungsträgern

- Weiterentwicklung von verbindlichen Vereinbarungen der Zusammenarbeit im Bereich der Eingliederungshilfe für junge Menschen $(\mathbb{S} 35 \mathrm{~s}$ SGB VIII)
"Gerade bei Kindern und Jugendlichen mit besonderen Unterstützungsbedarf kann es in Übergangssituationen zu Krisen kommen«

der Weiterentwicklung der Förderschulen konnten konkrete, gemeinsam erarbeitete Kooperationsansätze entwickelt werden, wie etwa der "Runde Tisch" - eine gemeinsame Fallbesprechung von Kolleginnen und Kollegen aus der Schule und dem Jugendamt - bis hin zu der aktiven Mitarbeit von Lehrerinnen und Lehrern aus der Förderschule bei
Ein weiteres Projekt erfolgt in der $\mathrm{Zu}$ sammenarbeit zwischen einer Herforder Grundschule, einer Herforder Realschule und der Jugendförderung. Hier werden wir ein gemeinsames Konzept erproben, indem eine sozialpädagogische Fachkraft Kinder für einige Monate im Übergang zwischen diesen beiden Schulen begleiten wird.

\section{Ausblick}

Wir wollen unseren »Herforder Weg" gemeinsam mit unseren Kooperationspartnern weiterverfolgen und die Tragfähigkeit immer wieder prüfen. Dazu gehört auch zu realisieren, was noch fehlt, wo der Weg noch verbessert werden kann, und wo er noch ausbaufähig ist.

Der Weg kann nur dann von allen angenommen werden, wenn die Kooperationen zwischen den Institutionen auch durch die beteiligten Menschen im Alltag gelebt und als hilfreich und unterstützend erlebt werden kann. In der Zusammenarbeit ist es immer wieder nötig, an gegenseitigen Vorurteilen und $\mathrm{zu}$ hohen Erwartungen zu arbeiten und das Herangehen und die Haltung des anderen in seiner Profession zu verstehen und zu akzeptieren.

Gleichwohl bleibt für das Jugendamt die gesetzliche Letztverantwortung im Einzelfall. Gleichwohl kann das Gesundheitssystem, das Bildungssystem als auch die freien Jugendhilfeträger das Kinn oder den Jugendlichen zeitweise oder vollständig aus ihren Angeboten ausschließen. Das Jugendamt bleibt kontinuierlicher Ansprechpartner für die Familien und die jungen Erwachsenen und hat den Auftrag auch in schwierigsten Situationen passgenaue Angebote vorzuhalten. Eine Delegation von Aufgaben des gesetzlichen Schulauftrages auf das Jugendamt, aber umgekehrt auch die Delegation von Aufgaben des Jugendhilfeträgers auf die Schule, kann nicht der Weg sein - eine Kooperation unterschiedlicher Professionen auf Augenhöhe ist Voraussetzung einer gelingenden Zusammenarbeit.

Wir wollen in Herford die Kinderund Jugendlichen in den Mittelpunkt unserer gemeinsamen Betrachtungen stellen und vertrauen auf die gemachten Erfahrungen in der Zusammenarbeit, auch für schwierige Aufgaben und Probleme adäquate Lösungen und Unterstützungen mit den jeweiligen Institutionen zu finden. 